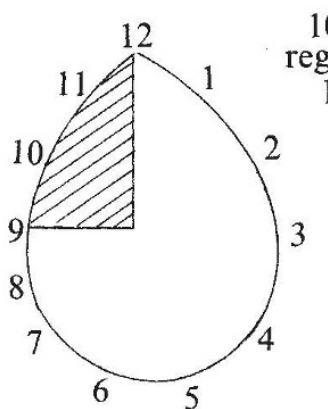

$10 \& 11$

egenerate
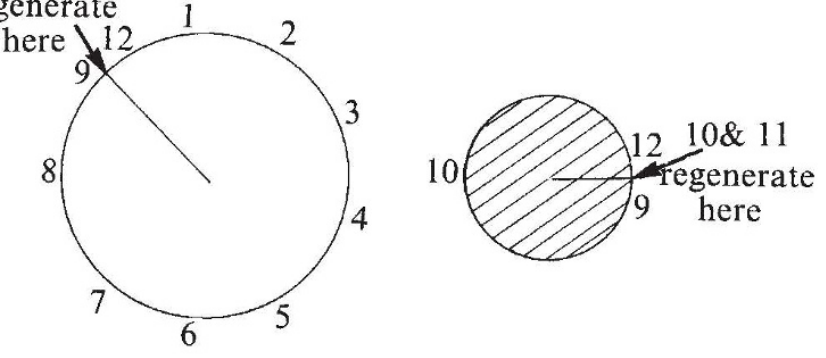

Fig. 2 The wing disk of Drosophila. The cut edges of the fragments come together and intercalary regeneration occurs between them.

can also account for the pattern of intercalary regeneration recently reported by Haynie and Bryant for Drosophila wing disk (Nature, 259, $659-662 ; 1976)$. If a quadrant is removed from the disk and the two fragments allowed to complete their development, the major fragment always regenerates the missing pattern and the minor one always mirror-duplicates the existing one. If the manner in which the cut edges heal is taken into account, intercalary regeneration of polar values accurately predicts this behaviour (Fig. 2). Bruce Carlson's experiments on the regeneration of multiple digits in axolotls give results which however do not entirely conform to the pattern seen in newt blastema. Carlson has explored the effects of rotating separately the different tissues of the stump and finds that the pattern of regeneration is distorted only when mesodermal tissues (muscle or dermis) are rotated. But whereas Bryant finds supernumerary limbs after either antero-posterior or dorso-ventral reversal of the blastema, Carlson obtains multiple digits only from anteroposterior rotation.

S. Strub (Zürich) reported what seems to have been the disruption of the proximo-distal, as opposed to the circumferential, coordinates of the Drosophila leg disk by disaggregation of a quadrant of the disk. Such fragments develop entirely distal structures. These are normally specified at the centre of the disk, where French, Bryant and Bryant suggest that the gradient is lowest; but how disaggregation should cause such sinking to a uniform low is not clear.

It was generally agreed that if such puzzles are to be solved by theoretical modelling, the models will have to be formulated in precise quantitative terms, both for the establishment of the morphogenetic gradient and for its interpretation by the cells. Richard Gardner (University of Oxford) pointed out that whereas for example the homoeotic mutants of Drosophila could be used to shed some light on the cellular response, mutants affecting the establishment of gradients are unknown. The best that models can do is to provide logically consistent quantitative descriptions of experimental phenomena, as long as the molecular mechanisms of morphogenesis remain inaccessible. It is reported that a British Forces team will shortly attempt Everest without oxygen.

\section{Inverse square law for gravity?}

\section{from a Correspondent}

'Every schoolboy knows' that gravitational attraction varies inversely as the square of the distance between the attracting masses-or at least it used to be common knowledge. Newton himself first gave strong support to the 'inverse square law' by comparing the rate at which the Moon accelerates towards the Earth with gravitational acceleration at the Earth's surface. He later provided compelling evidence by showing that Kepler's laws for the motion of the planets followed from the same law of attraction-that is, that they would move on ellipses with the Sun at one focus, and the square of their periods would be proportional to the cube of their major axes. Subsequent study of the motion of the planets and satellites has confirmed the truth of the inverse square law at astronomical distances (say from 1,000 $\mathrm{km}$ upwards) to a high degree of precision. It is therefore intriguing that in an article by D. R. Long in this issue of Nature (page 417), the question of the validity of the inverse square law at laboratory distances has been raised. An examination of the most accurate laboratory determinations of the constant of gravitation, $G$, suggests a systematic shift with the separation of the attracting masses-the larger the separation, the larger the value of $G$. In other words, Long suggests that the inverse square law is not valid, and that we have to add an additional short-range repulsion. The experiment reported here confirms his suggestion, by comparisons at distances of 4.5 and $30 \mathrm{~cm}$.

The question of a deviation from the inverse square law has been raised most seriously in 'recent' times by Newcomb, who showed that a small change in the form of the law could explain anomalies in the motion of Mercury. These anomalies are explained naturally by Einstein's General Theory of Relativity, which may be interpreted in a sense as predicting deviations from the inverse square law of gravitation. However, at laboratory distances with laboratory masses, Einstein's theory predicts that the inverse square law should hold to a high precision. Long's suggestion will therefore not meet with ready acceptance. If substantiated, the implications, for example, for our knowledge of the interiors of the Earth and the Sun would be considerable. We infer their masses from our knowledge of the constant of gravitation, and Long's experiment indicates that the limiting values of $G$ at large distances may be several percent higher than is presently accepted. Moreover the implications for a quantum theory of gravity would be serious.

One is tempted to echo the reaction of the bishop's wife to the suggestion that man is derived from the apes'let us hope that it is not true-or if it is, that it won't become generally known.' More responsibly, we should affirm our willingness to submit theory to experimental proof, and hope that Long's suggestion will stimulate a critical experimental appraisal. $\mathrm{He}$ has drawn attention to the remarkable fact that there is no accurate experimental evidence for the inverse square law of gravitation at laboratory distances. By comparison, very accurate null experiments on the basis first indicated by Cavendish have shown that the exponent in the inverse square law for electrostatics differs from two by less than $3 \times 10^{-16}$ (Williams, Faller, and Hill, Phys. Rev. Lett., 26, 721; 1971). Can anyone devise an accurate null experiment which will confirm (or deny) the inverse square law for gravitation?

\section{Erratum}

In the article 'Disappearing habitats' (Nature, 259, 365; 1975) the floral survey mentioned was carried out by $\mathrm{Mr}$ and $\mathrm{Mrs} \mathrm{C}$. Scotter and not by P. M. Wade. 